\title{
FISH is not Suitable as a Standalone Test for Detecting Fetal Chromosomal Abnormalities
}

\author{
Meena Lall $^{1} \cdot$ Surbhi Mahajan $^{1} \cdot$ Pushpa Saviour $^{1} \cdot$ Preeti Paliwal $^{1} \cdot$ \\ Anju Joshi $^{1} \cdot$ Nitika Setia $^{1} \cdot$ Ishwar C. Verma $^{1}$
}

Received: 29 April 2015/Accepted: 17 June 2015/Published online: 17 July 2015

(C) Society of Fetal Medicine 2015

\begin{abstract}
Karyotyping and fluorescence in situ hybridization (FISH) detect fetal chromosome abnormalities. The choice between karyotyping and FISH is still debatable. In a developing country, parents face an emotional and economic constraint of a prenatal test. Therefore, the results of karyotyping and FISH were analyzed to determine the percentage of clinically abnormal fetuses which would be missed by using standalone FISH. Amniotic fluid samples from 9033 high-risk pregnancies were subjected to karyotyping and FISH for chromosomes 13, $18,21, \mathrm{X}$, and Y. Karyotype and FISH were normal in 8680 $(96.1 \%)$ of these samples and $353(3.9 \%)$ had abnormal karyotypes: $285(3.2 \%)$ were aneuploidies, also detected by FISH and $68(0.7 \%)$ were structural chromosomal aberrations not detected by FISH. Out of these 68 structural aberrations, $40(0.4 \%)$ were balanced rearrangements with no apparent clinical significance and $28(0.3 \%)$ were unbalanced rearrangements with potential clinical significance. By standalone FISH, $0.3 \%$ clinically-significant samples would have been missed. A $0.2 \%$ risk of procedure-related abortion may be acceptable but a $0.3 \%$ risk of having an abnormal child may not be acceptable to the parents. FISH may be offered as a first test, followed by karyotyping. Although, karyotyping increases the cost, it is preferable to carry this out once an invasive procedure has been opted for, with its accompanying risk of miscarriage.
\end{abstract}

Meena Lall

lallmeena@gmail.com

1 Centre of Medical Genetics, Sir Ganga Ram Hospital, Rajender Nagar, New Delhi 110060, India
Keywords Karyotype · FISH - Fetal chromosomes · Aneuploidy · Prenatal

\section{Introduction}

Amniocentesis with subsequent karyotyping and targeted rapid aneuploidy detection (RAD) by fluorescence insitu hybridisation (FISH) was offered to pregnant women with positive biochemical screening test, advanced maternal age, abnormal ultrasound, previous child with Down syndrome or other congenital abnormalities. Parents who are referred for prenatal testing are always under emotional stress of bearing an abnormal child. In a developing country, most of these parents have to deal with not only emotional stress but also an economic constraint of the incurring costs of prenatal diagnostic procedures and the tests. Karyotyping is considered as the gold standard for prenatal diagnosis of numerical chromosomal abnormalities (aneuploidies) and structural chromosomal rearrangements [1]. But it has its pitfalls as it requires a great deal of technical expertise, tissue culture facilities, and a long waiting period ( 2 weeks), and is more expensive. The commonest chromosomal disorders, in which the fetus remains alive even though abnormal, are the aneuploidies of the chromosomes 13,18, 21, X, or Y.

FISH test is a targeted approach for detection of these aneuploidies. It does not require tissue cultures; results are quicker ( 2 days) and it is more cost effective. Keeping this in mind, RAD tests like FISH, quantitative fluorescence polymerase chain reaction (QF-PCR) [2, 3] or multiplex ligation-dependent probe amplification (MLPA) [4] are often preferred for faster results.

Therefore, the aim of the study was to conduct a retrospective analysis on the outcome of the pregnancies with 
abnormal karyotype and FISH test results, to recognize the clinically-significant chromosomal abnormalities which can be missed, if FISH was used as a standalone test. This would then help to decide that if both techniques are available, and if emotional stress and cost issues are also to be addressed, which would be the preferred test.

\section{Materials and Methods}

Amniotic fluid samples from 9080 pregnant women were referred for prenatal detection of chromosomal defects. Karyotyping and FISH test were done on 9033 samples. Remaining 47 were heterozygous parents or carriers of balanced translocations who were not offered FISH test, as only karyotyping can rule out chromosomal structural abnormalities. Therefore, the testing strategy in the present study was decided according to referral indications (Table 1).

Out of 9033 referrals (Table 1), 5590 (61.9\%) women screened positive for Down syndrome by biochemical test; $1390(15.4 \%)$ women had high maternal age; 1233 (13.6\%) women had abnormal ultrasound; 669 (7.4\%) had previous child with Down syndrome; 151 (1.7\%) had previous child with other congenital anomalies.

Amniotic fluid samples were cultured and processed for giemsa trypsin-giemsa (GTG) banding and karyotyping [5]. Both numerical and structural abnormalities were identified and classified using International System for Human Cytogenetic Nomenclature (ISCN), 2013 [6].

FISH [7] was performed using Vysis AneuVysion DNA probes on uncultured amniocytes, as targeted approach for RAD of the five common aneuploidies of chromosomes 13, 18, 21, X, and Y. Results of FISH were issued within 24-48 $\mathrm{h}$ and a full karyotype within 14-18 days of receipt of the amniotic fluid sample in the laboratory.

\section{Results}

Out of 9033 samples, $8680(96.1 \%)$ received normal FISH and normal karyotype results while 353 (3.9\%) received abnormal results (Table 2; Fig. 1). Out of these 353 chromosomal abnormalities, 285 (3.2\%) were numerical, all detected by karyotyping and FISH (Fig. 2). Trisomy 21 was the largest aneuploidy category comprising 195 $(68.4 \%)$ samples, $38(13.3 \%)$ had trisomy 18, $13(4.6 \%)$ had trisomy 13, 11 (3.9\%) were 47, XXY, 10 (3.5\%) were $47, \mathrm{XXX}$, four $(1.4 \%)$ were $\mathrm{XO}$, five $(1.8 \%)$ were $\mathrm{XO} / \mathrm{XX}$, two $(0.7 \%)$ were $\mathrm{XO} / \mathrm{XY}$, four $(1.4 \%)$ had triploidy and three $(1 \%)$ had double abnormality including aneuploidy. All 285 couples opted for termination of the pregnancies.

The remaining $68(0.7 \%)$ samples had structural abnormalities (Table 2; Fig. 1), which were detected only by karyotyping and not by FISH. Out of these $68(0.7 \%)$ structural chromosomal abnormalities, $40(0.4 \%)$ were apparently balanced, their pregnancies were continued and 28 $(0.3 \%)$ were unbalanced rearrangements, their pregnancies were terminated as per the decision taken by the parents.

\section{Discussion}

The results (Fig. 1) of karyotype and FISH were comparable in $96.1 \%$ (8680/9033) normal and $3.2 \%$ (285/9033) numerically-abnormal results. FISH test results were found to be $100 \%$ sensitive and $100 \%$ specific in all these 8965 women. Therefore, FISH as a standalone test proved to be effective as a rapid and cost-effective strategy in these $99.3 \%$ (8965/9033) amniotic fluid samples. FISH was not effective for the remaining $0.7 \%$ (68/9033) abnormal samples.

A retrospective analysis (Fig. 1) was done on all the 353 (3.9\%) abnormal results to review the outcome of these pregnancies to affirm their clinical significance. Aneuploidies were detected by karyotype and by FISH in 285 (3.2\%) of 9033 fetuses and all these 285 pregnancies were terminated by the parents, therefore the results were considered clinically significant. However, the remaining $0.7 \%$ (68/9033) samples which had structural chromosomal abnormalities were detected only by karyotype but missed by FISH. These were $19 \%$ (68/353) of the total chromosomal abnormalities which were missed by FISH.

Out of these $0.7 \%$ (68/9033) samples, $0.4 \%$ (40/9033) had structural rearrangements which were apparently balanced. The blood samples of all 40 parents were
Table 1 Testing strategy for karyotyping and FISH used in the present study

\begin{tabular}{llll}
\hline Indication for the test & $\begin{array}{l}\text { No. of referrals } \\
(\mathrm{n}=9080)\end{array}$ & FISH & Karyotyping \\
\hline Positive biochemical screening test & 5590 & Yes & Yes \\
High maternal age & 1390 & Yes & Yes \\
Abnormal ultrasound & 1233 & Yes & Yes \\
Previous child with trisomy 21 & 669 & Yes & Yes \\
Previous child with other congenital abnormalities & 151 & Yes & Yes \\
Heterozygous parents & 47 & No & Yes \\
\hline
\end{tabular}


Table 2 Out of 9033 amniotic fluid samples, 353 (3.9\%) had abnormal results

\begin{tabular}{|c|c|c|c|}
\hline \multirow{2}{*}{$\frac{\text { Indications }}{(\mathrm{n}=9033)}$} & \multirow{2}{*}{$\begin{array}{l}\text { Numerical aberrations } \\
(\mathrm{n}=285)(3.2 \%)\end{array}$} & \multicolumn{2}{|c|}{ Structural aberrations $(\mathrm{n}=68)(0.7 \%)$} \\
\hline & & Balanced $(\mathrm{n}=40)(0.4 \%)$ & Unbalanced $(\mathrm{n}=28)(0.3 \%)$ \\
\hline \multirow{20}{*}{$\begin{array}{l}\text { Positive biochemical screening } \\
\text { test }(5590)(61.9 \%)\end{array}$} & $+13(\mathrm{n}=5)$ & $46, \mathrm{t}(1 ; 4)$ & $2 q-$ \\
\hline & $+18(\mathrm{n}=21)$ & $46, \mathrm{t}(1 ; 4)$ & $3 p+$ \\
\hline & $+21(\mathrm{n}=98)$ & $46, \mathrm{t}(1 ; 10)$ & $9 q-$ \\
\hline & $\mathrm{XXX}(\mathrm{n}=1)$ & $46, \mathrm{t}(2 ; 5)$ & $46, \mathrm{t}(13 ; 21)$ \\
\hline & $\mathrm{XXY}(\mathrm{n}=4)$ & $46, \mathrm{t}(2 ; 6)$ & $\mathrm{Xp}-$ \\
\hline & $\mathrm{XO}(\mathrm{n}=1)$ & $46, \mathrm{t}(3 ; 8)$ & $\mathrm{Xq}-$ \\
\hline & $\mathrm{XO} / \mathrm{XX}(\mathrm{n}=2)$ & $46, \mathrm{t}(3 ; 13)$ & $\mathrm{i}(\mathrm{Xq})(\mathrm{n}=4)$ \\
\hline & $\mathrm{XO} / \mathrm{XY}(\mathrm{n}=2)$ & $46, \mathrm{t}(7 ; 9)$ & $+\operatorname{mar}(\mathrm{n}=3)$ \\
\hline & $69, X X Y(n=2)$ & $46, \mathrm{t}(7 ; 13)$ & \\
\hline & & $46, \mathrm{t}(9 ; 18)$ & \\
\hline & & $45, \mathrm{t}(13 ; 22)$ & \\
\hline & & $45, \mathrm{t}(13 ; 14)(\mathrm{n}=6)$ & \\
\hline & & $45, \mathrm{t}(14 ; 21)$ & \\
\hline & & $45, \mathrm{t}(21 ; 21)(\mathrm{n}=2)$ & \\
\hline & & $\operatorname{Inv}(2)(\mathrm{n}=2)$ & \\
\hline & & Inv (5) & \\
\hline & & Inv (6) & \\
\hline & & $\operatorname{Inv}(8)(\mathrm{n}=3)$ & \\
\hline & & Inv (19) & \\
\hline & Total $=136$ & Total $=28$ & Total $=13$ \\
\hline \multirow[t]{7}{*}{ High maternal age (1390) (15.4\%) } & $+13(\mathrm{n}=3)$ & $46, \mathrm{t}(2 ; 6)$ & $6 q+$ \\
\hline & $+18(\mathrm{n}=7)$ & $46, \mathrm{t}(11 ; 22)$ & $7 q-$ \\
\hline & $+21(\mathrm{n}=38)$ & & $13 \mathrm{p}+$ \\
\hline & $\mathrm{XXX}(\mathrm{n}=5)$ & & $\mathrm{iXq}$ \\
\hline & $\mathrm{XXY}(\mathrm{n}=2)$ & & $+\operatorname{mar}$ \\
\hline & $\mathrm{XO}(\mathrm{n}=2)$ & & \\
\hline & Total $=57$ & Total $=2$ & Total $=5$ \\
\hline \multirow[t]{11}{*}{ Ultrasound abnormality (1233) (13.6 \%) } & $+13(\mathrm{n}=5)$ & $46, \mathrm{t}(2 ; 4)$ & $3 q-$ \\
\hline & $+18(\mathrm{n}=10)$ & $45, \mathrm{t}(13 ; 21)(\mathrm{n}=2)$ & $5 p-$ \\
\hline & $+21(\mathrm{n}=55)$ & $46, \mathrm{t}(14 ; 22)$ & $9 \mathrm{p}+$ \\
\hline & $\mathrm{XXX}(\mathrm{n}=4)$ & Inv (2) & $19 p+$ \\
\hline & $\mathrm{XXY}(\mathrm{n}=3)$ & Inv $(8)$ & $20 \mathrm{q}+$ \\
\hline & $\mathrm{XO}(\mathrm{n}=1)$ & Inv (11) & $22 \mathrm{q}+$ \\
\hline & $\mathrm{XO} / \mathrm{XX}(\mathrm{n}=3)$ & & $\mathrm{i}(\mathrm{Xq})$ \\
\hline & $48, \mathrm{XYY},+21$ & & + mar \\
\hline & $69, \mathrm{XXX}(\mathrm{n}=1)$ & & \\
\hline & $69, \mathrm{XXY}(\mathrm{n}=1)$ & & \\
\hline & Total $=84$ & Total $=7$ & Total $=8$ \\
\hline \multirow[t]{4}{*}{ Previous trisomy 21 (669) (7.4\%) } & $+21(\mathrm{n}=4)$ & $45, \mathrm{t}(13 ; 21)$ & $6 \mathrm{q}+$ \\
\hline & $\mathrm{XXY}(\mathrm{n}=2)$ & $45, \mathrm{t}(15 ; 21)$ & \\
\hline & $47, \mathrm{t}(12 ; 21),+21$ & & \\
\hline & Total $=7$ & Total $=2$ & Total $=1$ \\
\hline \multirow[t]{2}{*}{ Previous congenital anomalies (151) (1.7\%) } & $47, \mathrm{t}(21 ; 21),+$ mar & $\mathrm{t}(1 ; 11)$ & $\operatorname{Der}(4) \mathrm{t}(4 ; 18)$ \\
\hline & Total $=1$ & Total $=1$ & Total $=1$ \\
\hline
\end{tabular}


Fig. 1 Flow chart showing all results

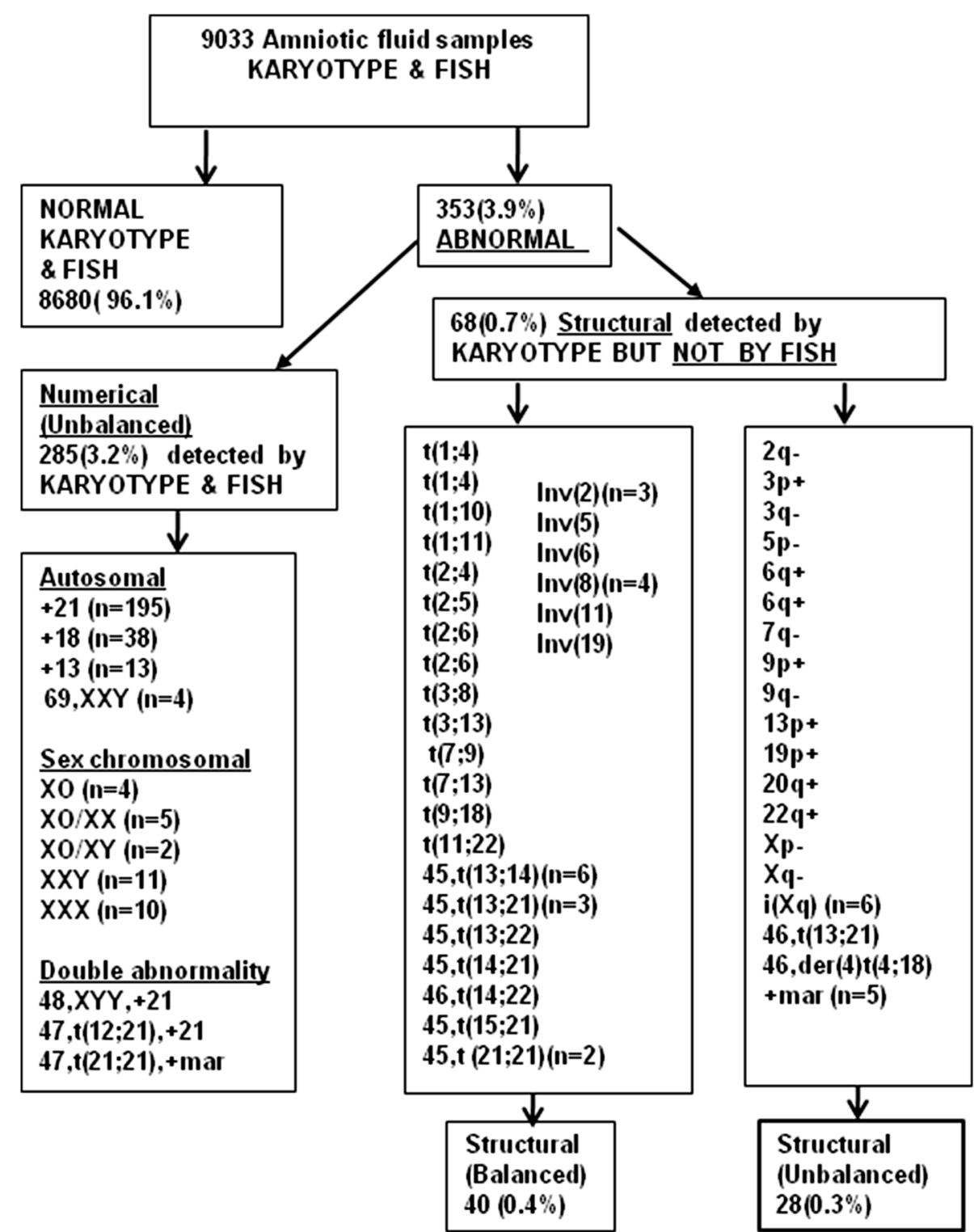

karyotyped and one parent out of each of the 40 couples was a balanced translocation carrier like the fetus. They all opted to continue their pregnancies, even though they were counseled about a small chance of gene disruption at the point of chromosome breakage and reunion in the translocation. Therefore, the balanced rearrangements in the fetus were concluded as potentially-insignificant results.

The remaining $0.3 \%$ (28/9033) structural rearrangements were unbalanced and could result in an abnormal phenotype, therefore, these 28 parents decided to terminate the pregnancies. Therefore, $0.3 \%$ was the residual results of chromosomal abnormalities with risk of clinical significance which were not detected by FISH. These were $8 \%$ (28/353) of the overall chromosome abnormalities detected only by karyotype.
Trisomy 13, 18, and 21 together accounted for $87 \%$ (249/285) of the total aneuploidies and sex chromosome aneuploidies were $11 \%(32 / 285)$ of the total aneuploidies (Fig. 1). Sex chromosomal abnormalities may have milder phenotypes but these are not accepted by Indian parents, therefore all pregnancies with sex chromosomal abnormalities were also terminated. The UK National Health Services (NHS) and the UK National Screening Committee (NSC) (2004) recommended that to cut down cost of prenatal diagnosis in the UK, RAD tests for chromosomes 13, 18, and 21 would be performed. The sex-chromosome aneuploidies were excluded from these tests. The women, who test positive by the screening programs, are offered RAD test as a standalone approach to rule out aneuploidies. In response to this decision by the NHS and NSC, Caine et al. [8] from the Association of Clinical Cytogeneticists 
(ACC), conducted a large survey on 119,528 amniotic fluid and 23077 chorionic villus samples and showed that about $1 \%$ of all prenatal samples would have a chromosomal abnormality which would go undetected by FISH and $33 \%$ of these abnormalities would be of clinical importance. These $33 \%$ abnormalities included the sex chromosomal and balanced translocations in their study. Nevertheless, a joint statement by the American College of Medical Genetics and the American Society for Human Genetics [9] reaffirmed that all RAD-test results must be followed-up with karyotyping. There is still no consensus on the most cost-effective strategy that should be implemented to diagnose affected fetuses in prenatal testing. As described by Hills et al. [2] and Ogilvie et al. [10] required staffing levels are substantially higher for a full karyotype service compared to a QF-PCR only as an aneuploidy detection service. We observe that all these are views from the West, but a developing country like India has a different perspective on this scenario. Such as, we do not have a National Health Scheme which would influence the overall budget of the health department. Having an economicallyvaried population, some parents will go all the way, but some may not. Also, all Indians would abort a fetus with sex-chromosome abnormalities, which may not be the case in the West.

Limiting prenatal testing to any rapid detecting technique such as FISH, QF-PCR, or MLPA would result in fetuses with karyotype abnormalities proceeding to detection at anomaly scan or through to term, resulting in late termination or the live birth of infants with congenital abnormalities, with the associated medical, emotional, and financial consequences. To avoid this, some strategy has to be formed for prenatal testing. About $80 \%$ of antenatal karyotypes are generated by antenatal screening programs [8]. Therefore, the indication of the test or reason for referral may also be taken into consideration to choose between karyotyping and FISH. Although some authors [11] have reviewed samples mainly from categories where screening tests were positive for Down syndrome, the present study has reviewed all referrals to form a strategy. Six groups can be formed according to the indication of the prenatal test (Table 1): Mothers who have screened positive for biochemical marker tests, mothers with high maternal age (above 35 years), mothers with abnormal ultrasound of the fetus, mothers with a previous child with common aneuploidy, and mothers with previous child with congenital defects or chromosomal abnormalities other than aneuploidies and heterozygous parents.

Heterozygous parents (Table 1), with balanced translocation should be offered only karyotyping as the structure of the particular chromosomes involved in their balanced translocation have to be examined carefully. When major congenital abnormalities are detected during routine ultrasound analysis, traditional karyotyping should be preferred to look for chromosomal structural abnormalities, to help in management of future pregnancies. For the referral group with previous history of congenital or structural chromosomal abnormality, other than aneuploidy, favorable test would be karyotyping.

Eventually, the only three referral groups such as women with high maternal age, biochemical screening test positive, and previous child with aneuploidy may consider FISH test for RAD. However, in the present study, besides the aneuploidies that were detected, $68 \%$ (19/28) unbalanced structural chromosomal anomalies were also found in the samples of these three referral groups (Table 2): $46 \%(13 / 28)$ in samples of women who screened positive in the biochemical tests, $18 \%(5 / 28)$ in the category with high maternal age, and $4 \%(1 / 28)$ in the category with previous child with aneuploidy. Therefore, structural chromosomal anomalies can occur in any group. Such results would be considered as incidental or unexpected results, for which, the couple should be counseled prior to making a choice of the tests. It is the presence of these infrequent unexpected results in prenatal tests which make the rapid aneuploidy approach debatable [11]. Pregnant women who benefit from amniocentesis also take a $0.2 \%$ risk of a procedure-related miscarriage. Therefore, additional information of such unexpected diagnosis of chromosomal anomaly detected by karyotyping would reduce the anxiety and economic constraint caused by the birth of an abnormal child with clinically-significant chromosomal abnormalities.

Finally, to summarize, FISH may be quick and cost effective, but has its advantages and disadvantages. It is a quick test but it has a targeted approach to rule out only the targeted five common aneuploidies of chromosomes 13, 18, $21, \mathrm{X}$, and Y. FISH was found to be $100 \%$ sensitive and $100 \%$ specific for the detection of the targeted aneuploidies and has the capacity to detect triploidies, as found in the present study and also reported by others [12]. However, aneuploidies of other chromosomes and structural chromosomal abnormalities can be detected only by karyotyping. Occasionally, low-level mosaicism is encountered, for which karyotyping may validate some results. In discolored or blood-stained samples subjected to FISH, maternal-cell contamination cannot be ruled out, if the fetus is not a male. Other available methods for RAD, as quick as FISH are QF-PCR [2, 3] and MLPA [4]. Sparks et al. [12] reviewed these other available RAD tests. They reported the sensitivity and specificity of QF-PCR to be $95.65 \%$ and $99.97 \%$ and that of MLPA as $100 \%$ and $99.8 \%$, respectively.

A joint statement by the American College of Medical Genetics and Genomics (ACMG) and American Society of Human Genetics (ASHG), 2000 [9] has also stated that 
Fig. 2 Distribution of aneuploidies $(\mathrm{n}=285)$

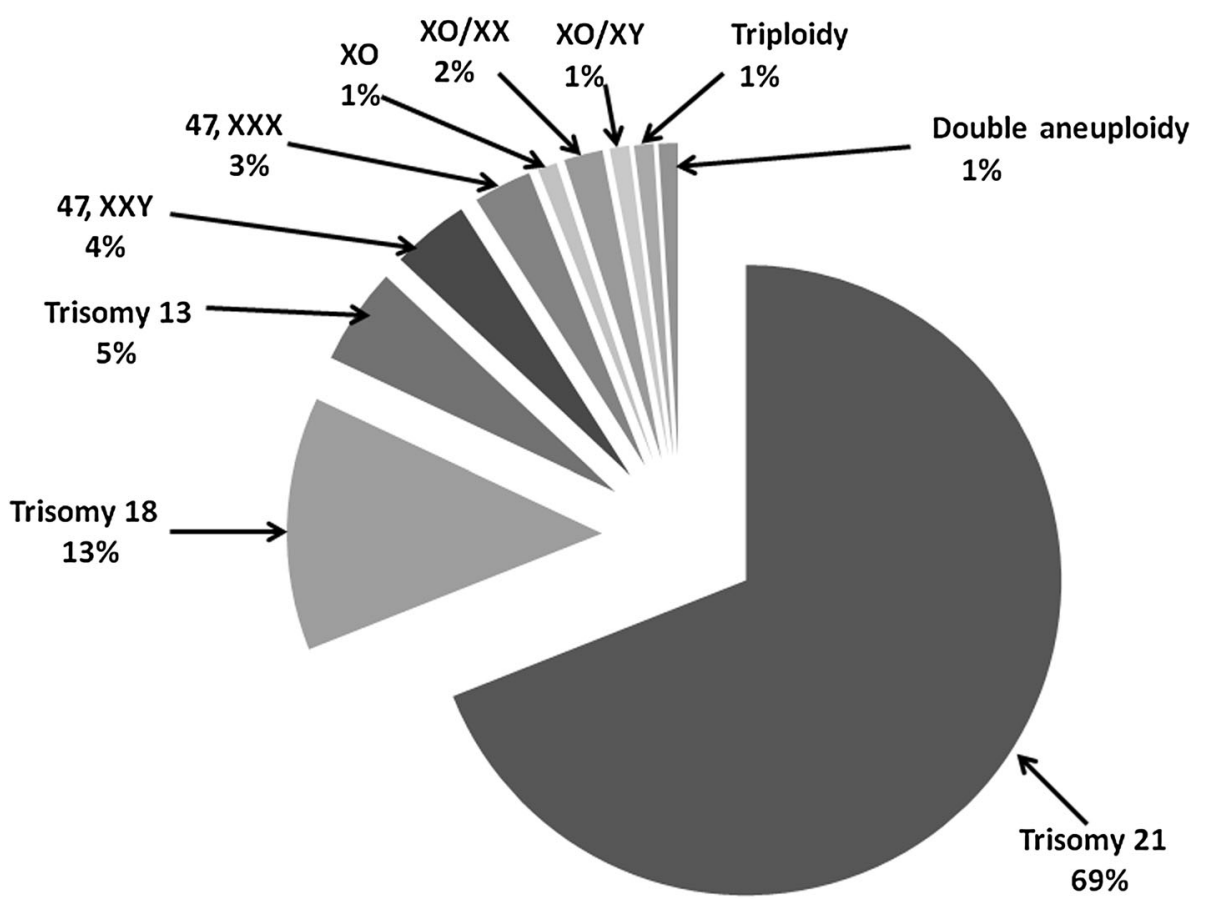

FISH results may not detect the structural abnormalities but the targeted aneuploidies can be reported immediately. However, irreversible action based on positive results should be carefully considered along with other medical evidence currently used in making prenatal diagnosis. Therefore, FISH should be used as adjunct with any other prenatal procedures or tests such as ultrasound or karyotyping. Based on the data of the present study, we concur with this statement, as unexpected results can occur in all categories of test indications. Pretest genetic counseling is essential for prenatal tests, so that informed consent is given by the couple referred for the test. Generally, the accepted aim of prenatal testing is to offer opportunities for autonomous reproductive choice [13].

Microarray $[14,15]$ is a newer test which is being evaluated by laboratories for its feasibility and cost effectiveness for prenatal diagnosis. This test has the potential of detecting chromosomal abnormalities from the whole genome at a higher resolution than karyotyping and the results can be obtained earlier. If costs can be taken care of, this could replace karyotyping, FISH, QF-PCR, and MLPA. Expertise for interpretation of these results can be developed.

Noninvasive prenatal testing (NIPT) $[16,17]$ is another newer test which uses cell-free fetal DNA from maternal plasma to screen fetal aneuploidy. This is being offered by external commercial agencies but this also is being used to detect the targeted five common aneuploidies and it is going through introspection by governing authorities due to prevention of sex determination in India. A patient with a positive NIPT test result is referred for genetic counseling and is offered invasive prenatal diagnosis for confirmation of the result.

\section{Conclusion}

FISH alone is quick and cost effective, but it is targeted to rule out aneuploidies of chromosomes 13, 18, 21, X, and Y. To identify other chromosome aneuploidies and structural anomalies, karyotyping is essential. Pretest genetic counseling to explain this is essential as for the couple every pregnancy is precious. However, the advantage of FISH, if used as an adjunctive test, is that the initial tension of both, the parents and the physician, would be reduced. Although, karyotyping increases the cost, it is preferable to carry this out, once an invasive procedure has been opted for, with its accompanying risk of miscarriage.

Compliance with ethical standards

Conflict of interest None.

\section{References}

1. Bui TH. Prenatal cytogenetic diagnosis: gone FISHing, BAC soon! Ultrasound Obstet Gynecol. 2007;30:247-51.

2. Hills A, Donaghue C, Waters J, et al. QF-PCR as a stand-alone test for prenatal samples: the first 2 years'experience in the London region. Prenat Diagn. 2010;30:509-17. 
3. Cirigliano V, Voglino G, Ordonez E, et al. Rapid prenatal diagnosis of common chromosome aneuploidies by QF-PCR, results of 9 years of clinical experience. Prenat Diagn. 2009;29:40-9.

4. Van Opstal D, Boter M, de Jong D, Van den Berg C, Bruggenwirth HT, Hajo IJ. Rapid aneuploidy detection with multiplex ligation-dependent probe amplification: a prospective study of 4000 amniotic fluid samples. Eur J Hum Genet. 2009;17:112-21.

5. Priest JH. Prenatal chromosome diagnosis and cell culture. In: Margaret JB, editor. The ACT cytogenetics laboratory manual. New York: Raven press; 1991. p. 149-204.

6. Shaffer LG, McGowan-Jordon J, Schmid M, editors. An international system for human cytogenetic nomenclature. Basel: S. Karger; 2013.

7. Feldman B, Aviram-Goldring A, Evans MI. Interphase FISH for prenatal diagnosis of common aneuploidies. In: Fan Y-S, editor. Molecular cytogenetics. New Jersey: Humana press; 2002. p. $219-42$.

8. Caine A, Maltby AE, Parkin CA, et al. Prenatal detection of Down's syndrome by rapid aneuploidy testing for chromosomes 13, 18, and 21 by FISH or PCR without a full karyotype: a cytogenetic risk assessment. Lancet. 2005;366:123-8.

9. American College of Medical Genetics. Technical and clinical assessment of fluorescence in situ hybridisation: an ACMG/ ASHG position statement. 1. Technical considerations. Genet Med. 2000;2(6):356-61.

10. Ogilvie CM, Lasswood A, Chitty L, et al. The future of prenatal diagnosis: rapid testing or full karyotype? An audit of chromosome abnormalities and pregnancy outcomes for women referred for Down syndrome testing. BJOG. 2005;112:1369-75.

11. Leung WC, Lau ET, Lau WL, et al. Rapid aneuploidy testing (knowing less) versus traditional karyotyping (knowing more) for advanced maternal age: what would be missed, who should decide? Hong Kong Med J. 2008;4:6-13.

12. Sparkes R, Johnson J, Langlois S, et al. New molecular techniques for the prenatal detection of chromosomal aneuploidy. J Obstet Gynaecol Can. 2008;301(617-21):622-7.

13. de Jong A, Dondrop WJ, Timmermans DRM, et al. Rapid aneuploidy detection or karyotyping? Ethical reflection. Eur J Hum Genet. 2011;19(10):1020-102.

14. Shaffer L, Coppinger J, Alliman S, et al. Comparison of microarray-based detection rates for cytogenetic abnormalities in prenatal and neonatal specimens. Prenat Diagn. 2008;28:789-95.

15. Carey L, Scott F, Murphy K, et al. Prenatal diagnosis of chromosomal mosaicism in over 1600 cases using array comparative genomic hybridization as a first line test. Prenat Diagn. 2014;34:478-86.

16. Wang JC, Sahoo T, Schonberg S, et al. Discordant noninvasive prenatal testing and cytogenetic results: a study of 109 consecutive cases. Genet Med. 2015;17:234-6.

17. Quezada MS, Gil MM, Francisco C, et al. Screening for trisomies 21,18 and 13 by cell-free DNA analysis of maternal blood at 10-11 weeks' gestation and the combined test at 11-13 weeks. Ultrasound Obstet Gynecol. 2015;45(1):36-41. 